\title{
Synthesis of azamacrocycle stabilized palladium nanoparticles: Controlled size and one-dimensional growth
}

\author{
JEYARAMAN ATHILAKSHMI and DILLIP KUMAR CHAND* \\ Department of Chemistry, Indian Institute of Technology Madras, Chennai 600036, India \\ e-mail: dillip@iitm.ac.in
}

\begin{abstract}
Palladium nanoparticles (PdNPs) of uniform size in the range of 3-5 nm are prepared in $\mathrm{MeOH}$ and $\mathrm{MeCN}: \mathrm{MeOH}$ by solvent-assisted reduction of palladium acetate in the presence of a hexaazamacrocyclic ligand, L1. For the mixed solvent system different ratio of the solvents was tried i.e., 1:1, 1:3 and 3:1. In all cases the concentration and ratio of Pd(II) to $\mathbf{L 1}$ was maintained at $2 \mathrm{mM}: 1 \mathrm{mM}$. In another experiment NPs were prepared in $\mathrm{MeOH}$ by lowering the concentration of $\mathbf{L 1}$ to $0.5 \mathrm{mM}$, where chain-like assemblies of PdNPs was observed. Importantly, the solutions are found to be stable at RT for months.
\end{abstract}

Keywords. Azamacrocycle; stabilizer; palladium nanoparticles; chain formation; TEM micrographs; UV-vis; powder XRD.

\section{Introduction}

Size and shape of materials at nanoscopic level can modulate the related physical and chemical properties greatly. ${ }^{1}$ Synthesis of metal nanoparticles in a controlled manner is thus attractive. Palladium nanoparticles (PdNPs) of different sizes are reported where chemical reduction of palladium salts such as $\mathrm{PdCl}_{2},{ }^{2}$ $\mathrm{Pd}(\mathrm{OAc})_{2}{ }^{3}$ and $\mathrm{Pd}\left(\mathrm{NO}_{3}\right)_{2}{ }^{4}$ were performed in solution phases by external reducing agents such as inorganic hydrides, ${ }^{2}$ citrate and ascorbate. ${ }^{5}$ Synthesis of the nanoparticles are also performed by reduction of $\mathrm{Pd}(\mathrm{II})$ in the solvents such as polyols or alcohols ${ }^{6}$ by heating or under ultrasonic treatment. In these methods suitable stabilizers are employed to prevent coagulation of the NPs. Recently, the use of supramolecules such as calixarenes, ${ }^{7}$ porphyrins ${ }^{8}$ and cyclodextrins ${ }^{9}$ and some other bulky organic ligands ${ }^{10}$ are exploited as stabilizers for PdNPs. Such large molecules provide steric stabilization thereby hindering the interaction of particles approaching one another and produce smaller size particles. We have reported ${ }^{11}$ the synthesis of PdNPs in $\mathrm{MeCN}: \mathrm{MeOH}$ mixture in the absence of external reducing agent and stabilizers where $\mathrm{MeOH}$ is the reducing agent and the co-solvent $\mathrm{MeCN}$ acted as stabilizer. However, the particles are not uniform in size $(\sim 20$ to
$50 \mathrm{~nm}$ ) and appeared aggregated as seen in TEM micrographs. These particles showed tendency of coagulation when heated or allowed to stand for longer time. In this work, we inspected the role of a hexaazamacrocyclic ligand, L1 (figure 1) aiming at uniform sized and stable PdNPs.

\section{Experimental}

\subsection{Materials}

All the glass vials are rinsed with aqua regia followed by distilled water and dried. Palladium acetate (99.9\%) was obtained from Aldrich whereas AR grade $\mathrm{MeOH}$ and $\mathrm{MeCN}$ were obtained from SRL and used as received. The hexaazamacrocyclic ligand $\mathbf{L 1}$ was synthesized as mentioned in the literature. ${ }^{12}$

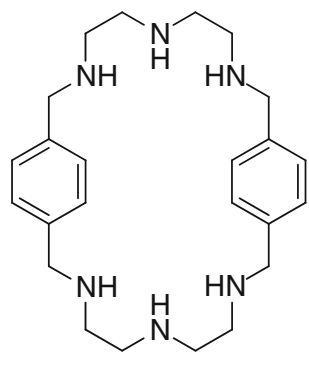

L1 


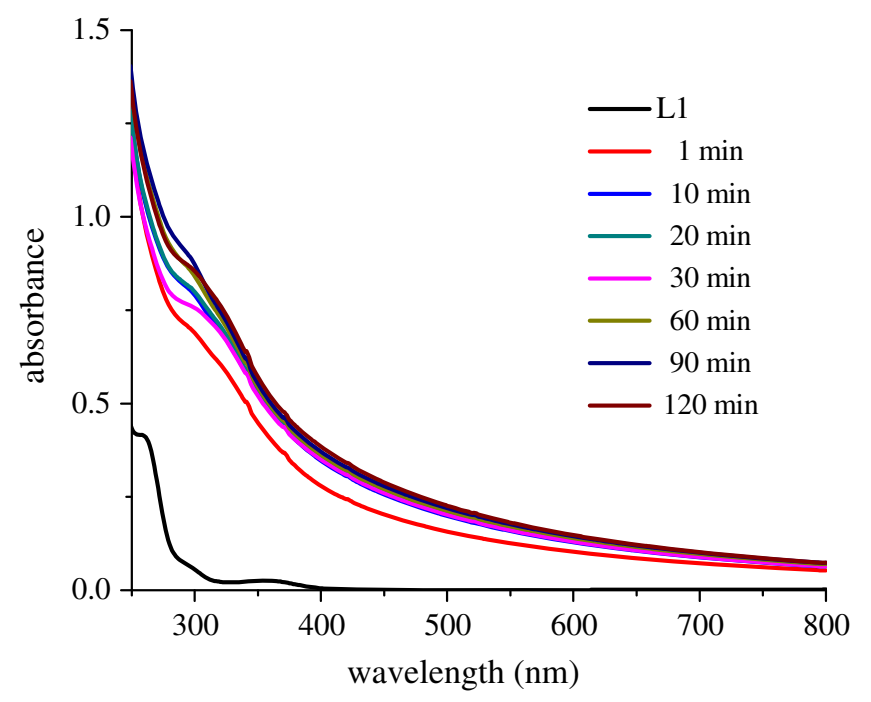

Figure 2. Representative UV-vis spectra of $2 \mathrm{mM}$ $\mathrm{Pd}(\mathrm{OAc})_{2}$ in $\mathrm{MeCN}: \mathrm{MeOH}(1: 1)$ in the presence of $1 \mathrm{mM}$ of $\mathbf{L 1}$ monitored at 1, 10, 20, 30, 60, 90 and $120 \mathrm{~min}$. The solution was heated at $60^{\circ} \mathrm{C}$ and spectra are recorded at $30^{\circ} \mathrm{C}$.

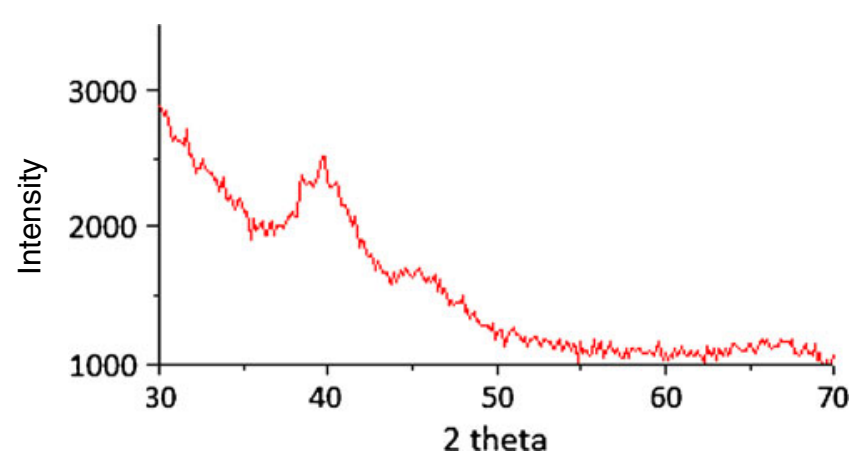

Figure 3. Powder XRD pattern of the PdNPs sample as obtained from the entry 2 of table 1 (Reference: JCPDS No. 89-4897).

\subsection{Characterization techniques}

All UV-vis absorption spectra were recorded using a JASCO V-550 UV-vis spectrophotometer at room temperature. UV-vis absorption of each solution was measured in a $3 \mathrm{~mL}$ quartz cuvette over the wavelength range 200 to $800 \mathrm{~nm}$. High-resolution TEM micrographs were recorded using a Tecnai G-20, a $200 \mathrm{KeV}$ TEM operating at an accelerating voltage of $200 \mathrm{KeV}$. TEM samples were prepared by dropping colloidal solution of nanoparticles onto a carbon coated copper grid followed by air drying at room temperature. XRD studies of the samples were carried out on a XD-D1 Shimadzu Horizontal Diffractometer using Copper $\mathrm{K} \alpha$ radiation as the source $(\lambda=1.5406 \AA)$. The samples were scanned in the $2 \theta$ range of $20-80^{\circ}$. The samples were prepared by evaporation of the solvent under reduced pressure and air drying. About $10 \mathrm{mg}$ of a dried sample was fixed with the help of grease on to a thin-glass plate and diffracted.

\subsection{Synthesis of PdNPs in the presence of $\mathbf{L} \mathbf{1}$}

To $50 \mathrm{~mL}$ of hot methanol $\left(60^{\circ} \mathrm{C}\right), \mathbf{L 1}(20.5 \mathrm{mg}$, $0.05 \mathrm{mmol}$ ) was added. Heating was continued for another $30 \mathrm{~min}$ followed by addition of $\mathrm{Pd}(\mathrm{OAc})_{2}$ $(22.44 \mathrm{mg}, 0.1 \mathrm{mmol})$ to the hot solution. The colour of the solution got changed from yellow to brownish black within $10 \mathrm{~min}$. The formation of PdNPs was confirmed by UV-vis spectroscopy and transmission electron microscopy. The prepared sample was cooled to RT and the solution was evaporated under reduced pressure and dried under vacuum to obtain the sample as powder. Similar procedure was followed for other solvents systems.

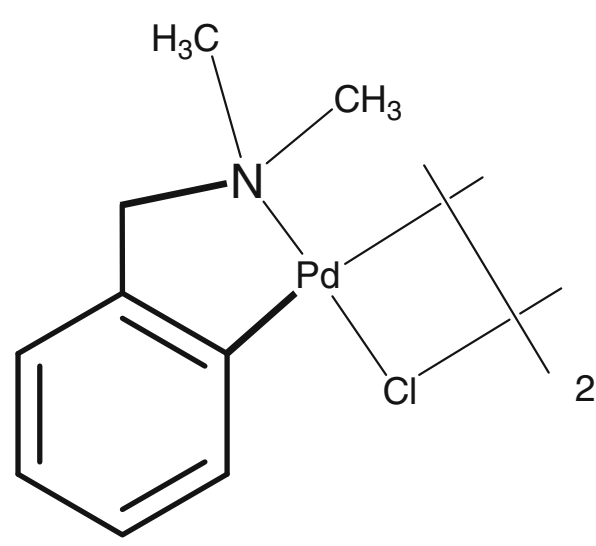

(a)<smiles>c1cc2ccc1CNCCNCCNCc1ccc(cc1)CNCCNCCNC2</smiles>

L1

(b)

Figure 4. A comparative structure of (a) cyclopalladated compound with (b) L1. 


\section{Results and discussion}

Preparation of PdNPs by reduction of $\mathrm{Pd}(\mathrm{II})$ using $\mathrm{MeOH}$ as reductant in the absence or presence of a cosolvent, however, in the presence of a stabilizer is
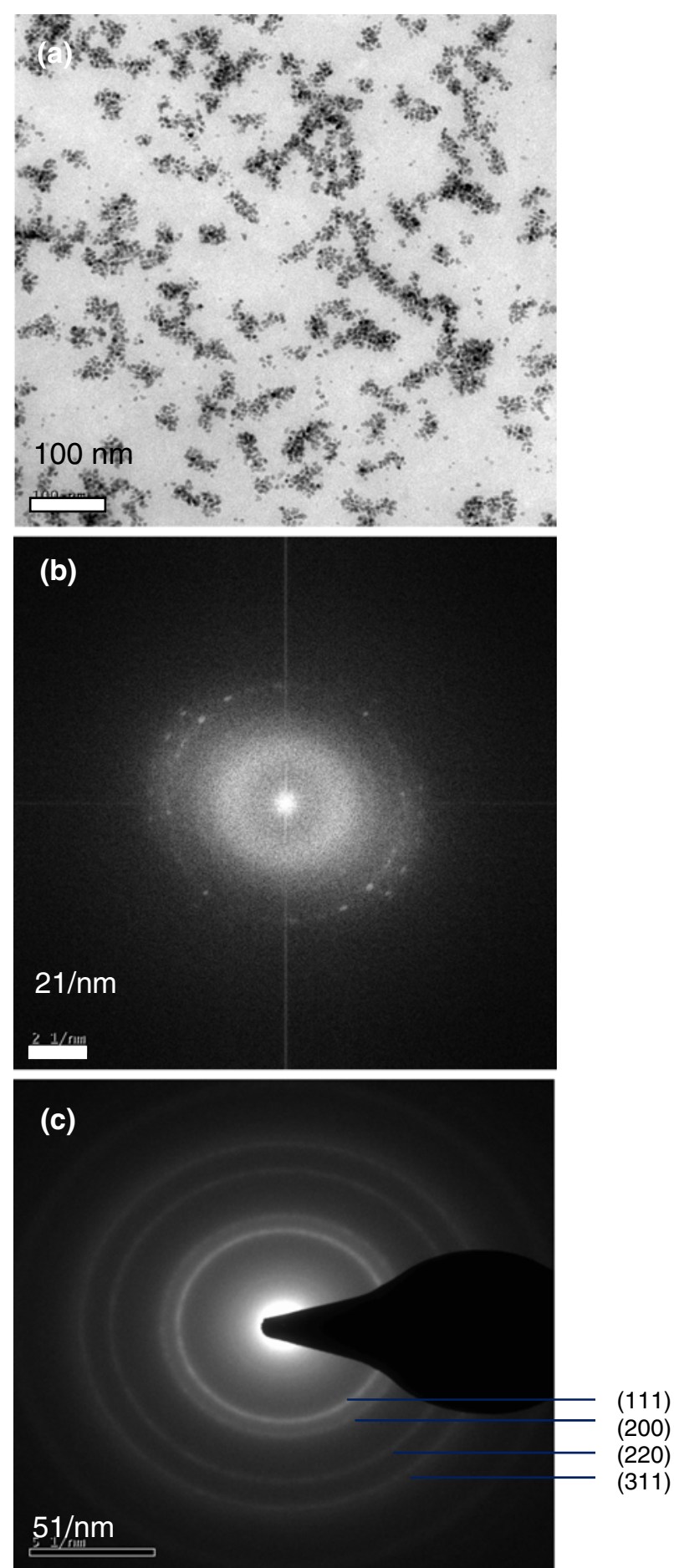

Figure 5. (a) TEM image of PdNPs obtained from $2 \mathrm{mM}$ solution of palladium acetate in $\mathrm{MeOH}$ in the presence of $1 \mathrm{mM} \mathrm{L1}$ at $60^{\circ} \mathrm{C}$, (b) FFT image, (c) SAED image. possible. ${ }^{13}$ Stabilization of PdNPs were achieved by a variety of amine based protecting agents such as lauryl amine,${ }^{14}$ octyl amine ${ }^{15}$ and oleyl amine. ${ }^{16}$ Stabilization of silver nanoparticles by $\mathbf{L} \mathbf{1}$ is reported by us ${ }^{17}$
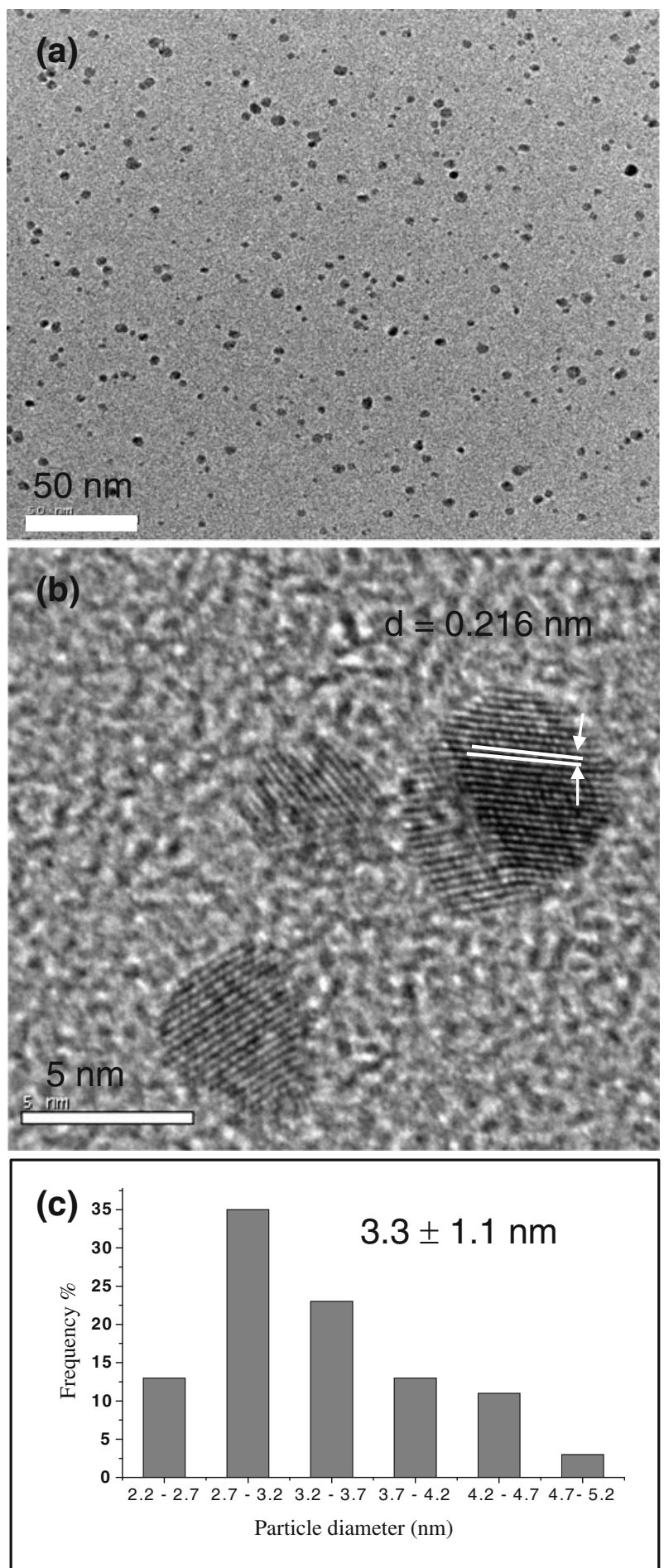

Figure 6. (a) TEM, (b) HRTEM image and (c) particle size distribution plot of PdNPs obtained from $2 \mathrm{mM}$ palladium acetate in 1:1 ratio of $\mathrm{MeCN}$ : $\mathrm{MeOH}$ mixture with $1 \mathrm{mM} \mathbf{L 1}$ at $60^{\circ} \mathrm{C}$. 
thus opening an avenue of further use of $\mathbf{L 1}$ in stabilization of other metal nanoparticles. We have now prepared hexaazamacrocycle, L1 stabilized PdNPs from palladium acetate using methanol as reducing agent in the absence or presence of $\mathrm{MeCN}$. In the absence of a stabilizer although reduction happens with in a few minutes at $60^{\circ} \mathrm{C}$ to generate PdNPs the particles are readily coagulated in $\mathrm{MeOH}$ medium and with time in $\mathrm{MeOH} / \mathrm{MeCN}$ medium. In this work, the PdNPs are prepared in $\mathrm{MeOH}$ and also in three different mixtures of $\mathrm{MeCN}$ : $\mathrm{MeOH}(1: 1,1: 3$ and 3:1). In all cases the concentration and ratio of $\mathrm{Pd}(\mathrm{II})$ to $\mathbf{L 1}$ is maintained at $2 \mathrm{mM}: 1 \mathrm{mM}$. In another experiment the NPs were prepared in $\mathrm{MeOH}$ by lowering the concentration of $\mathbf{L 1}$ to $0.5 \mathrm{mM}$ where upon chain-like assemblies of PdNPs was observed. Importantly, the solutions are found to be stable at RT for months.

The formation of PdNPs was followed by recording their UV-vis spectra. It is well-known that MNPs absorb photons in UV-vis region due to coherent oscillation of the conduction band electrons induced by the interacting electro-magnetic fields. However, PdNPs do not show pronounced peak due to surface plasmon. We also observed a comparable pattern in the UV-vis spectra, and hence infer the formation of PdNPs. The usual peaks of Pd(II) around $400 \mathrm{~nm}$ disappeared due to formation of PdNPs. Representative UV-vis spectra of a sample prepared in $\mathrm{MeCN}: \mathrm{MeOH}$ (1:1) is shown in figure 2.

The PdNPs could be isolated in solid state (as given in experimental section) by evaporation of the solvent. Powder XRD was taken for a representative sample prepared in $\mathrm{MeCN}: \mathrm{MeOH}(1: 1)$ in order to confirm the formation of NPs. As shown in figure 3, XRD pattern peaks are observed at 40,46 and $68^{\circ}$ which correspond to (111), (200) and (220) planes of fcc lattice, respectively. This finding indicates that PdNPs has fcc structure.

When the reduction of a metal salt is carried out in the presence of a ligand it is possible that during initial stages coordination complexes may form followed by chemical reactions that lead to NPs. A variety of Pd-organometallic precursors ${ }^{18}$ used as precatalysts in some organic transformations resulted in the rapid change of the colour of the solutions to black suggesting the formation of $\operatorname{Pd}(0)$. Structural studies by TEM revealed the presence of the nano-sized particles. Therefore a probable structure relevant to $\mathbf{L} \mathbf{1}$ was chosen by comparing the literature ${ }^{18 b}$ as shown in figure 4.

TEM images were obtained (figures 5-9) for all the solutions and the data is collected in table 1. It is found
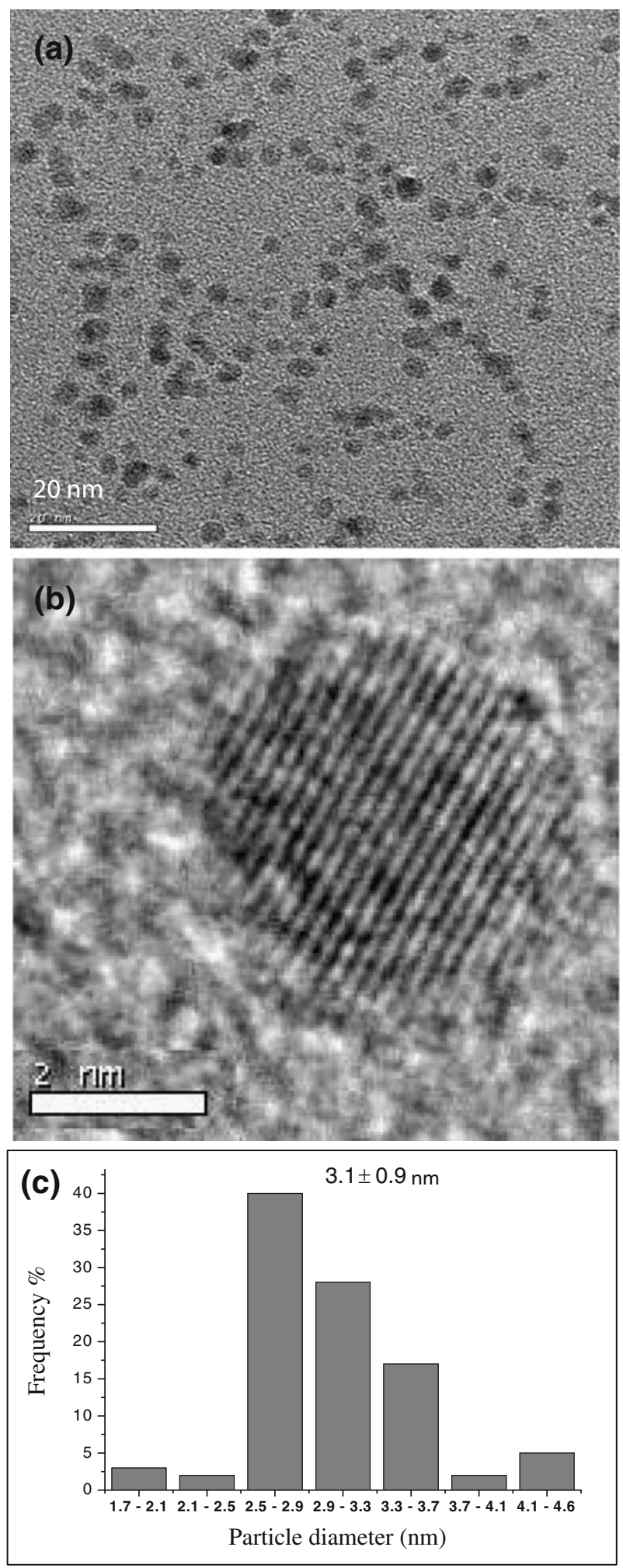

Figure 7. (a) TEM, (b) HRTEM image and (c) Particle size distribution plot of PdNPs obtained from $2 \mathrm{mM}$ palladium acetate in 1:3 ratio of $\mathrm{MeCN}: \mathrm{MeOH}$ mixture in the presence of $1 \mathrm{mM} \mathrm{L1}$ at $60^{\circ} \mathrm{C}$. 

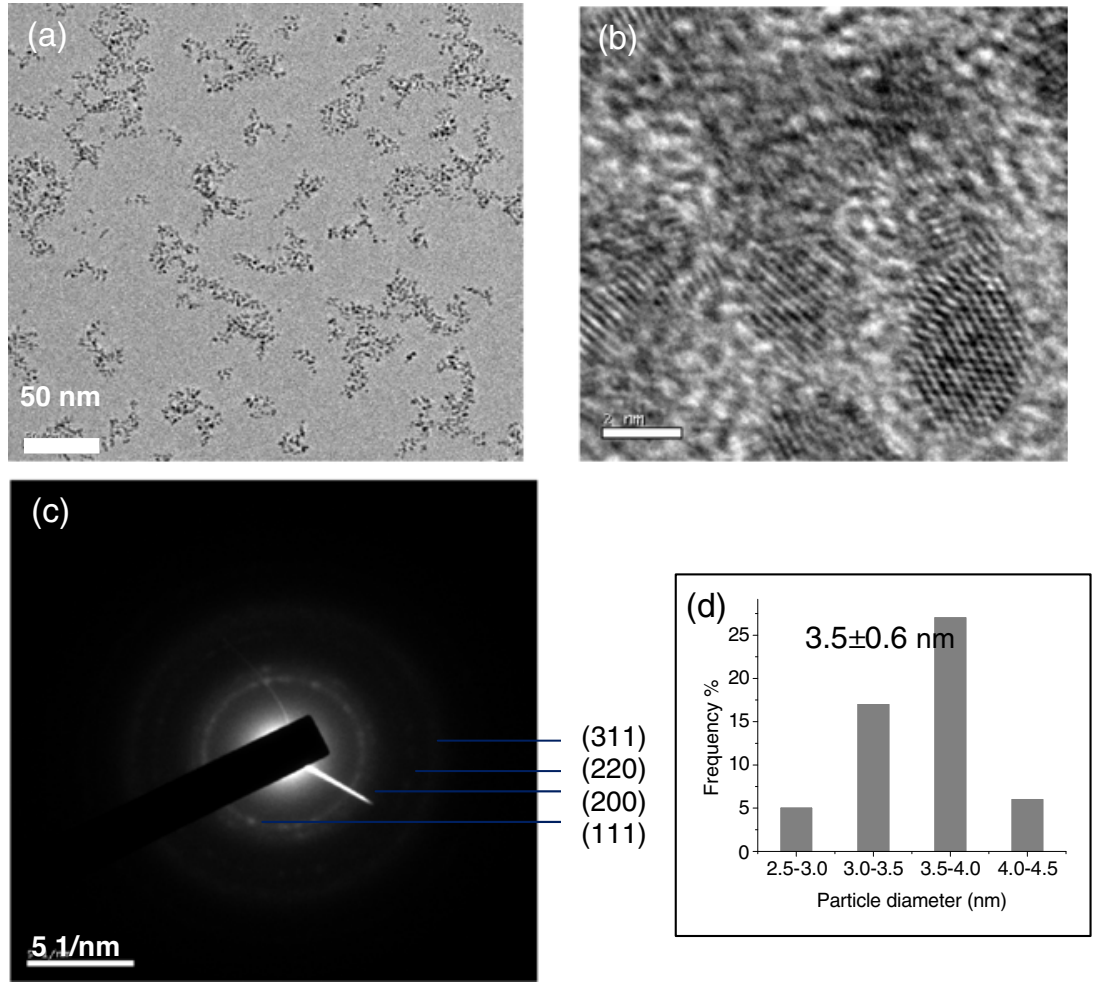

Figure 8. (a) TEM, (b) HRTEM image, (c) SAED image and (d) particle size distribution plot of PdNPs obtained from $2 \mathrm{mM}$ palladium acetate in 3:1 ratio of $\mathrm{MeCN}: \mathrm{MeOH}$ mixture in the presence of $1 \mathrm{mM}$ macrocycle, $\mathbf{L} \mathbf{1}$ at $60^{\circ} \mathrm{C}$.
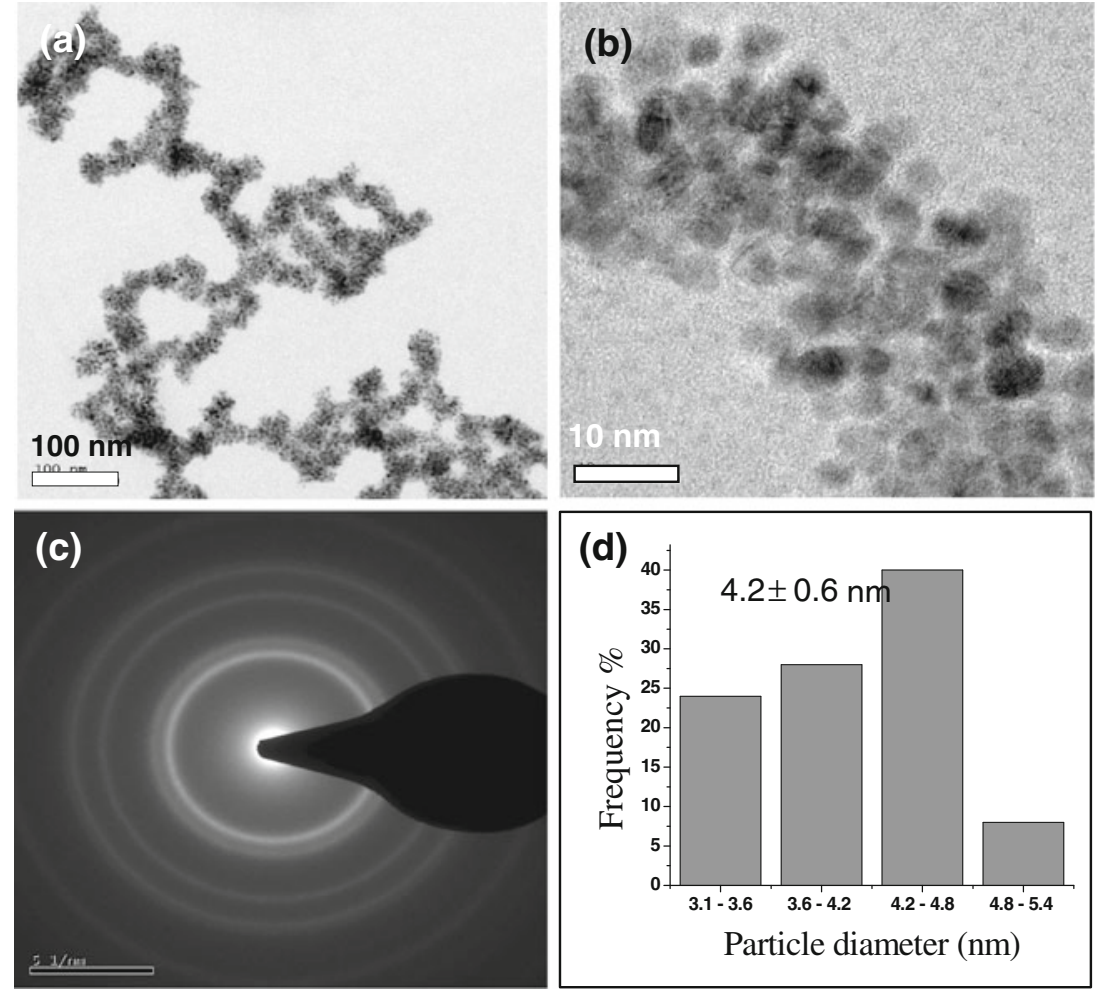

Figure 9. (a) TEM, (b) HRTEM image, (c) SAED image and (d) particle size distribution plot of $\mathrm{Pd}$ nanochains obtained from $2 \mathrm{mM}$ palladium acetate in the presence of $0.5 \mathrm{mM}$ of macrocycle, $\mathbf{L 1}$ in methanol at $60^{\circ} \mathrm{C}$. 
Table 1. Size of PdNPs calculated from TEM data for the samples prepared at $2 \mathrm{mM}$ concentration of palladium acetate precursor.

\begin{tabular}{lclcc}
\hline Entry & Ratio of Pd:L1 & Solvent(s) & Particle size and SD (nm) & TEM image \\
\hline 1 & $2: 1$ & MeOH & $5.0 \pm 1.2$ & Figure 5 \\
2 & $2: 1$ & MeCN: MeOH (1:1) & $3.3 \pm 1.1$ & Figure 6 \\
3 & $2: 1$ & MeCN: MeOH (1:3) & $3.1 \pm 0.9$ & Figure 7 \\
4 & $2: 1$ & MeCN: MeOH (3:1) & $3.5 \pm 0.6$ & Figure 8 \\
5 & $2: 0.5$ & MeOH & $4.2 \pm 0.6$ & Figure 9 \\
\hline
\end{tabular}

that the size of the particles varies from 3 to $5 \mathrm{~nm}$ and hence the distribution may be considered narrow and particles are of almost uniform size. Electron diffraction pattern of the PdNPs is shown in figures. The values of interplanar distance $\mathrm{d}_{h k l}$ calculated from the diffraction rings are in good agreement with the literature. ${ }^{19}$ This indicates the existence of face centred cubic structure of palladium in the NPs.

In the medium of methanol and by lowering the concentration of $\mathbf{L 1}$ to $0.5 \mathrm{mM}$ it appears that individual NPs are interlinked to one another as a chain which is observed via TEM and HRTEM images (figure 8). The affinity of nitrogen centres in $\mathbf{L 1}$ is apparently responsible for such design and it is also reported for PdNPs prepared in the presence of amine. ${ }^{20}$ Individual NPs are attached to one another causing agglomeration of the PdNPs to worm-like structures favoured in the medium of methanol. In the medium of $\mathrm{MeCN}: \mathrm{MeOH}$ with L1, MeCN produces high monodispersed particles by hindering the interaction of the particles along with L1.

In the absence of any stabilizers, PdNPs are precipitated when prepared in $\mathrm{MeOH}$. On the other hand, the presence of a coordinating co-solvent $\mathrm{MeCN}$ could prevent agglomeration of particles. However, the presence of a stabilizer e.g., $\mathbf{L 1}$ as discussed in this work, could result uniform sized PdNPs due to steric stabilization. When the amount of L1 with respect to the concentration of Pd is lowered presumably some of the macrocycle units are involved in capping adjacent particles thus resulting a chain-type arrangement. Without proper and detailed study further discussion on mechanistic aspect of chain-like assembly formation is not credible at this stage.

\section{Conclusion}

A simple method for the preparation of uniform sized palladium nanoparticles in the narrow range of 3 to $5 \mathrm{~nm}$ is described in this work. The growth of particles beyond this size is controlled by the steric bulk pro- vided by a hexaazamacrocyclic ligand which acted as a stabilizer. Reduction of palladium acetate to elemental palladium could be possible by reducing nature of the amine groups crafted in the macrocycle and also assisted by the known reducing agent $\mathrm{MeOH}$ which was the solvent of choice. While the addition of a co-solvent $\mathrm{MeCN}$ did not make much of a difference to the size of PdNPs, especially in $\mathrm{MeOH}$ medium when the concentration of the ligand is lowered it appears that individual NPs are interlinked with one another giving a chain-like arrangement. The materials are characterized by UVvis, powder XRD, TEM and HRTEM techniques. Use of the present ligand studied and other related ligands is expected to contribute richly toward the preparation of various metal nanoparticles.

\section{Acknowledgements}

We thank Council of Scientific and Industrial Research (CSIR), India for providing financial support (No.01 (2483)/11/EMR-II) to our research project on 'Functional noble metal nanoparticles'.

\section{References}

1. (a) Roduner E 2006 Chem. Soc. Rev. 35 583; (b) El-Sayed M A 2001 Acc. Chem. Res. 34257

2. Jana N R, Wang Z L and Pal T 2000 Langmuir 162457

3. Nemamcha A, Rehspringer J-L and Khatmi D 2006 J. Phys. Chem. B 110383

4. Mizugaki T, Murata M, Fukubayashi S, Mitsudome T, Jitsukawaa K and Kaneda K 2008 Chem. Commun. 241

5. Xiong Y, McLellan J M, Yin Y and Xia Y 2007 Angew. Chem. Int. Ed. 46790

6. Sun Y, Zhang L, Zhou H, Zhu Y, Sutter E, Ji Y, Rafailovich M H and Sokolov J C 2007 Chem. Mater. 192065

7. Huc V and Pelzer K 2008 J. Colloid Interface Sci. 3181

8. Imahori H, Kashiwagi Y, Endo Y, Hanada T, Nishimura Y, Yamazaki I, Araki Y, Ito O and Fukuzumi S 2004 Langmuir 2073

9. Senra J D, Fernando L, Malta B, da Costa H M, Michel R C, Aguiar L C S, Simas A B C and Antunes O A C 2009 Adv. Synth. Catal. 3512411 
10. (a) Schwarze M, Keilitz J, Nowag S, Parapat R Y, Haag R and Schomcker R 2011 Langmuir 27 6511; (b) Cargnello M, Wieder N L, Canton P, Montini T, Giambastiani G, Benedetti A, Gorte R J and Fornasiero P 2011 Chem. Mater. (dx.doi.org/ $10.1021 / \mathrm{cm} 2014658)$

11. Athilakshmi J, Ramanatha S and Chand D K 2008 Tetrahedron Lett. 495286

12. Chen D and Martell A E 1991 Tetrahedron 476895

13. (a) Arul Dhas N and Gedanken A 1998 J. Mater. Chem. 8 445; (b) Tanaka H, Koizumi S, Hashimoto T, Itoh H, Satoh M, Naka K and Chujo Y 2007 Macromolecules 40 4327; (c) Ornelas C, Salmon L, Aranzaes J R and Astruc D 2007 Chem. Commun. 4946; (d) Naka K, Itoh H and Chujo Y 2002 Nano Lett. 21183

14. Rao C R K, Lakshminarayanan V and Trivedi D C 2006 Mater. Lett. 603165
15. Yang Z, Klabunde K J and Sorensen C M 2007 J. Phys. Chem. C 11118143

16. Mazumder V and Sun S 2009 J. Am. Chem. Soc. 131 4588

17. Athilakshmi J and Chand D K 2010 Tetrahedron Lett. 516760

18. (a) Grigg R, Zhang L, Collard S, Ellis P and Keep A 2004 J. Organomet. Chem. 689 170; (b) Huang R and Shaughnessy K H 2006 Organometallics 25 4105; (c) de Vries J G 2006 Dalton Trans. 421

19. Navaladian S, Viswanathan B, Varadarajan $T$ K and Viswanath R P 2009 Nanoscale Res. Lett. 4181

20. (a) Naoe K, Kataoka M and Kawagoe M 2010 Colloids Surf. A 364 116; (b) Li Z, Gao J, Xing X, Wu S, Shuang S, Dong C, Paau M C and Choi M M F 2010 J. Phys. Chem. C 114723 